Mots. Les langages du politique

\title{
Un discours politique au féminin. Le projet d'Olympe de Gouges
}

\section{Jürgen Siess}

\section{OpenEdition}

\section{Journals}

Édition électronique

URL : https://journals.openedition.org/mots/293

DOI : $10.4000 /$ mots.293

ISSN : 1960-6001

Éditeur

ENS Éditions

\section{Édition imprimée}

Date de publication : 1 juillet 2005

Pagination : 9-21

ISBN : 2-84788-080-1

ISSN : 0243-6450

\section{Référence électronique}

Jürgen Siess, "Un discours politique au féminin. Le projet d'Olympe de Gouges », Mots. Les langages du politique [En ligne], 78 | 2005, mis en ligne le 31 janvier 2008, consulté le 22 avril 2022. URL : http:// journals.openedition.org/mots/293; DOI : https://doi.org/10.4000/mots.293 


\section{Un discours politique au féminin. Le projet d'Olympe de Gouges}

Sans doute les gender studies et la nouvelle histoire de la Révolution ont-ils contribué à conférer à Marie-Olympe de Gouges le statut de grande républicaine et féministe avant la lettre, mais aussi d'avocate de l'égalité1. En revanche, on a prêté moins d'attention à la spécificité de sa parole propre dans le contexte des discours révolutionnaires. Aussi le présent article tâchet-il, en s'inspirant des méthodes de l'analyse du discours, de faire ressortir cette part spécifique dans quelques textes marquants écrits entre 1788 et 1793. II s'agit de montrer dans quelle mesure Olympe de Gouges infléchit ou module le discours politique dominé par les hommes pour le subvertir à ses fins propres, réclamer les droits de la femme et promouvoir l'égalité des sexes.

Plus précisément, je voudrais analyser la façon dont les textes politiques d'Olympe de Gouges, en se coulant dans des genres divers, élaborent son discours relatif à la différence des sexes. Je retiendrai trois textes qui relèvent de genres de discours différents: $1 /$ la lettre ouverte ${ }^{2}, 2$ / l'épître dédicatoire, $3 /$ l'avis public. Chacun d'eux est lié à un but précis et à une situation spécifique. La Lettre au Peuple propose un impôt volontaire pour contribuer à la solution de la grave crise économique. L'épître adressée à la reine est censée servir la promotion d'une nouvelle Déclaration des droits qui parait à un moment où les femmes ont leurs propres clubs politiques. Dans l'avis intitulé Olympe de Gouges au Tribunal révolutionnaire, diffusé sous forme d'affiche à un moment où l'on s'apprête à dissoudre les clubs de femmes, elle s'adresse à une cour de justice exclusivement composée d'hommes: on verra qu'Olympe se réfère ici tout autant à son action publique qu'à son statut privé.

1. Citons, à titre d'exemple, Zamore et Mirza ou l'heureux naufrage, pièce contre l'esclavage des Noirs (1784, créée en décembre 1789).

2. Le terme n'existe pas encore à l'époque, et longtemps on ne fait pas de distinction nette entre « lettre publique » et « lettre ouverte» (voir M. Riot-Sarcey, 1998, «La lettre publique : les pétitions de femmes sous les monarchies constitutionnelles», Christine Planté éd., p. 221-232). Il se stabilise comme terme générique («Article de journal ou opuscule, généralement de caractère polémique, rédigé sous forme de lettre ", Trésor de la langue française, 1983) dans le dernier tiers du $19^{\mathrm{e}}$ siècle, ce sens se distinguant désormais clairement de celui de lettre non cachetée ou enfermée dans une enveloppe.

Université de Caen, THL, jsiess@noos.fr 


\section{JÜRGEN SIESS}

Si l'appel à l'acte patriotique, la sollicitation de soutien ou le plaidoyer s'effectuent dans un but politique, ils semblent cependant sous-tendus par un projet qui vise à l'établissement d'un rapport autre entre les deux sexes, à l'instauration de l'égalité. En analysant ces trois textes, il faudra donc dégager les modalités selon lesquelles chaque genre est repris et subverti, afin de voir dans quelle mesure le discours «égalitaire» en gestation se manifeste à travers le discours politique et quelle part il prend selon la situation et le dispositif choisi.

\section{Lettre au peuple}

Ce texte, publié par Olympe à la fin de l'année 1788, reprend le genre de la lettre publique - ancêtre de la lettre ouverte - pour lequel elle choisit le format et le support de la brochure ${ }^{3}$. Le titre principal semble désigner le public des lecteurs dans son ensemble; il joue toutefois sur l'ambivalence de «peuple nation » et «peuple condition sociale». Le second titre, ou Projet d'une caisse patriotique, accentue, quant à lui, l'intention de soumettre un plan d'intervention civique pour le bien commun.

Dans cette adresse au public, la locutrice se présente comme agissant seule, en «simple particulière » mais aussi comme faisant partie des «bons Citoyens » et, implicitement, du petit nombre de vrais « sages » que compte la nation (Écrits politiques 1, 1993, p. 37). Et, mettant l'accent sur l'argument politique, elle affirme être légitimée à parler en qualité de patriote. Cette légitimation semble nécessaire pour faire approuver son projet d'un impôt volontaire, moyen qu'elle propose pour étancher la dette de l'État et, partant, pour aider «l'Ouvrier [qui] manque de pain » (ibid., p. 38). L'interaction qu'un «membre du parti le plus faible» initie par la Lettre est motivée par le sens de la responsabilité civique, le zèle patriotique (p. 39). Le premier but est de se faire écouter par les citoyens malheureux que la locutrice invite à se détourner des " séditieux extravagants », et d'être bons pères. Il précède la présentation d'un projet destiné à redresser la situation de cette fraction particulière du peuple mais aussi de la nation entière (p. 41). Olympe entend « enflammer de l'amour de la patrie» tous les rangs de la société, sans exclure le roi qui a promis de veiller au bonheur de ses sujets, ni la reine à laquelle Olympe suggère de faire des économies sur ses dépenses (p. 43-44).

3. Le texte a paru le 12 décembre 1788 (0. Blanc, 2003, p. 106). Un compte rendu très favorable lui est consacré à la une du Journal général de France dès le 6 novembre 1788. Il sera beau, y lit-on, de suivre dans la carrière du législateur les femmes « qui, enflammées de l'amour de la patrie, l'exciteront ou le réveilleront de toutes parts, sinon par leurs écrits, du moins par leurs discours». 
Dans le corps de la lettre, on relève des invocations à des instances allégoriques qui, pour un temps, se substituent au premier allocutaire: "Ô vérité sublime! qui m’a toujours guidée» (p. 39), "Ô bonté paternelle qui doit enflammer le cœur de tout bon Français» (p. 40). L'adresse à la vérité - «Ôtemoi les moyens d'écrire, si jamais je peux trahir ma conscience éclairée par ta lumière» - fait suite à une captatio benevolentiæ destinée à légitimer la démarche de la locutrice : «Vous, Public [...], pourriez-vous me blâmer du motif qui m'encourage et qui ranime mes forces?» (p. 39) L'invocation à la vérité comme instance sublime est supposée infléchir le public en faveur de la locutrice, mais aussi rapprocher celle-ci de la Vérité, personnage placé au sommet, et ainsi faire participer la locutrice de la supériorité qui distingue la haute instance invoquée. Ensuite elle revient, pour se placer face à lui, à son allocutaire principal: au peuple exposé aux semeurs de trouble qui l'incitent à l'action violente (p. 40). Elle invite le peuple à déployer son énergie dans le travail qui profite à tous, à lui aussi bien qu'à la nation. Suit un nouveau changement de destinataire: à présent, la locutrice s'adresse à la Bonté. Louis XVI a fait preuve d'une clémence et d'une modération dignes d'admiration en réinstaurant dans sa fonction le ministre disgracié, homme vertueux et efficace. Aussi cette adresse à la figure de la Bonté prépare-t-elle la juxtaposition du père et du roi, la comparaison entre l'homme responsable de ses enfants et le roi veillant aux intérêts de son peuple. La locutrice fait ici entendre que les Français et Louis sont faits pour s'associer.

Dans la suite de son argument, la "Citoyenne zélée» entend s'inscrire comme troisième adhérente à cette association destinée à travailler pour le bien commun. On peut déceler, dans cette image de la citoyenne, une facette qui donne à voir la locutrice dans un rôle éminent: dans la mesure où celle-ci fait le lien entre le roi, dont elle se voit proche, et le peuple vers lequel elle se tourne, elle semble s'arroger une position qui, dans le champ du pouvoir, est réservée au roi. Et dans la conclusion de sa Lettre, elle réaffirme la légitimité de son entreprise : «Mon but est louable [...], rien ne peut me détourner du sentier que je me suis frayé », en faisant ressortir ses qualités particulières : cœur vrai, âme pure, caractère droit (p. 45), éléments qui confirment l'image de la «femme juste et sensible» qu'elle invoque peu avant. À présent, Olympe sollicite la prise de position du public considéré comme allocutaire compétent:

Ô Français! véritable(s) Français, connaissez mon âme toute entière : ce n'est point par ambition que j'écris cette épître; le bien seul de ma Patrie, et l'amour et le respect que j'ai pour mon Roi, ont seuls excité ma verve [...], je tairai mon nom [...], je craindrais de m'enorgueillir et d'empoisonner la simplicité dont la nature m'a douée. C'est avec des sentiments de fraternité que je suis pour tous mes compatriotes la plus zélée et la plus sincère Citoyenne. (p. 45)

La présentation de soi valorisante est à présent relativisée, aussi Olympe parvient-elle à un équilibre entre les deux images, de soi et de l'autre. 
Que peut-on relever dans le mode du dire? Olympe prétend que ses réflexions manquent d'énergie, que son style est diffus et décousu (p. 39). Cependant, l'emploi des règles de la rhétorique et le jeu avec les instances discursives qu'on observe dans son texte infirment ce propos. Rappelons le recours aux figures allégoriques et à la comparaison roi/père et l'emploi de la captatio benevolentiæ - il s'agit de trouver auprès du public l'approbation du projet politique et de légitimer l'objet de la lettre. Notons aussi l'emploi de l'ironie - «le style est plus naïf qu'éloquent»: présentant la naïveté comme un défaut imputable à la faiblesse du sexe féminin, Olympe joue sur un préjugé masculin. Il faut par ailleurs revenir au maniement de l'interrelation je-vous qui se situe dans le même contexte. Un jeu complexe avec les normes peut y être décelé. La locutrice semble placer le destinataire («Vous-Français») en face d'elle. Elle lui suggère de la suivre dans une entreprise initiée par elle mais faite pour servir ses intérêts à lui. Comme si elle voulait dire : si vous me suivez, le roi s'associera à vous et fera ce que vous souhaitez, c'est moi qui vais fonder l'association qui nous réunira tous les trois. Le jeu porte sur les instances discursives, l'adresse au roi manque, comme si la négociation décisive se faisait entre la Citoyenne zélée et le Peuple en détresse. Mais il y a plus. En plaçant sur le même plan le Public, la Vérité, la Bonté, le Peuple, la locutrice passe souverainement d'un allocutaire à l'autre. Ainsi l'énonciation manifeste une maitrise qui dément l'image de la femme modeste ou faible mise en place par l'énoncé.

Olympe recherche donc un subtil équilibre entre la sollicitation, qui comporte une part de modestie, et l'affirmation de soi qui la met en valeur par rapport à son destinataire principal. Le ton du plaidoyer ou de la proposition constructive prévaut aux dépens du ton offensif ou critique, et on ne trouve que quelques rares propos polémiques (ainsi, contre les écrivains qui s'intéressent aux grands et méprisent le peuple). L'initiative qu'elle prend et le fait qu'une femme soit à son origine paraissent trop audacieux pour pouvoir être acceptés par un éditeur sans de multiples précautions. Dès le début, l'écrivaine semble tenir compte des attentes de son public potentiel habitué aux formes consacrées du discours politique. Sans doute a-t-on, à l'époque, vu des brochures traitant de questions d'éducation ou d'éthique rédigées par des femmes, sans doute a-t-on accepté qu'elles s'approprient le discours pédagogique ou moral, mais le discours politique reste pour une large part l'apanage des hommes. Aussi Olympe, avant de révéler le point de vue féminin à partir duquel elle parle, donne-t-elle plusieurs références qui peuvent servir de garants - l'homme animal doué de raison, la sagesse du bon citoyen, le roi et, en tête, le «jugement naturel » (qui défie les préjugés, en particulier contre les femmes) : «C'est au Peuple que je m'adresse : je le prie de me lire avec attention et de juger si je pense en bonne Citoyenne. Sa Majesté, sans doute, ne trouvera point mauvais qu'une femme attendrie sur l'affliction générale, ose prévenir [...] des maux encore plus cruels » (p. 38). Et elle tâche de trouver une 
voie médiane dans laquelle hommes et femmes peuvent la suivre également: "Je suis un membre du Public à qui je soumets mes observations; mais un membre du parti le plus faible: si dans mes réflexions il n'y a point d'énergie, mon sexe m'en justifie» - on retrouve l'ironie (p. 38-39). C'est contre le préjugé à l'égard des femmes qu'elle argumente dans la conclusion de sa Lettre: "Les États-Généraux [...] ne pourront trouver déplacés les conseils d'une femme qui, en dépit de la légèreté naturelle à son sexe, n'en a pas moins de bonnes vues; ce sexe, qu'on se plait tant à accuser de frivolité, n'en a pas moins en général des idées souvent ingénieuses» - pour ajouter que les sages eux-mêmes en profitent quelquefois (p. 45). Par sa spirituelle affirmation que les femmes savent penser aussi bien que les hommes, Olympe revendique implicitement l'instauration de rapports d'égalité entre les deux sexes.

Est mise en valeur l'association fondée sur le sentiment qui réunit le peuple, le roi et la femme qui propose un projet politique en sollicitant l'engagement de ses deux partenaires. La situation du pays fournit à la femme de lettres l'occasion d'intervenir dans un domaine traditionnellement réservé aux hommes. La figure nouvelle de la femme politique ne s'arrête pas au rôle de simple associée ou médiatrice. Celle qui initie un projet visant à engager la nation entière prétend assumer une fonction qui semblait réservée à l'homme le plus haut placé : ainsi une position-clé est conférée à la femme 4 .

Dans ce texte, Olympe construit donc une image de soi susceptible de légitimer la prise de parole d'une femme dans les affaires publiques, matière qui n'est pas considérée de son ressort. La présentation de soi élève en même temps la femme digne de jouer un rôle politique et de remplir une fonction de médiation entre le roi et le peuple jusqu'à les diriger, tel un metteur en scène, vers une position qui la rend égale aux hommes.

\section{À la reine}

En 1791, Olympe fait précéder sa Déclaration des droits de la femme et de la citoyenne, que les rédacteurs de la première Déclaration risquent de rejeter, d'une adresse À la reine. Cette lettre, qui sollicite le soutien de MarieAntoinette, occupe la place de la traditionnelle épître dédicatoire. On verra cependant qu'au lieu de présenter ses respects à la dédicataire, l'auteur développe un argument politique à son intention. Un genre consacré est non seulement utilisé dans un sens singulier, mais aussi subverti à tous points de vue. Dès le début, l'épître, qui s'adresse à un membre de la famille royale, se

4. Ceci est confirmé par le frontispice dont Olympe est l'instigatrice: l'écrivaine y est placée au-dessus de Louis XVI et de Marie-Antoinette et tend son livre à celle-ci (voir l'illustration dans O. Blanc, 2003, p. 264). 
présente comme un hommage dépourvu du respect requis. Il est dès lors conséquent que la formule d'allocution choisie soit «Madame» et non, comme il se devait, «Majesté». Les premiers mots, «Peu faite pour [ce] langage », donnent à voir l'épistolière comme douée d'un esprit trop libre pour se soumettre aux conventions et se plaçant en dehors du monde de la cour. Aussi affirme-t-elle son intention de parler «franchement», opposant la parole sincère au langage courtois. Comme l'ont bien vu Christiane Veauvy et Laura Pisano (1997, p. 26), Madame de Gouges était fière de son langage direct et vrai qu'elle opposait elle-même au discours politique dominant.

L'image de soi que l'épistolière construit ensuite accuse les traits de la femme que caractérisent l'audace et l'énergie, et de la femme singulière qui se distingue par ses actes. Notons cependant qu'Olympe légitime la démarche insolite que constitue l'appel à la reine en évoquant un acte antérieur en faveur de celle-ci - «Je n'ai pas attendu [...] l'époque de la liberté: je me suis montrée avec la même énergie dans un temps où l'aveuglement des despotes punissait une si noble audace. Lorsque l'Empire vous accusait [...], j'ai eu la force de prendre votre défense. » Mais il y a plus. Olympe se réfère à cet autre acte en faveur de la destinataire comme s'il l'autorisait à prétendre au même rang que celle-ci. Et si les temps auxquels se situe l'écriture de la lettre sont propices à une initiative faite en toute liberté, Olympe sous-entend toutefois qu'elle a contribué elle-même à l'avènement de ces temps nouveaux. Elle semble se voir à la hauteur de la reine et occupant une position d'où elle peut lui parler de femme à femme. Si elle la met en valeur lorsqu'elle imagine sa destinataire dans la situation de la persécutée, elle ne juge pas moins la reine sur le comportement qu'elle va adopter à présent, au moment de prendre connaissance de la lettre. Aux yeux de Madame de Gouges, la figure de la reine persécutée appartient au passé; au moment où Olympe lui écrit, c'est la réaction de Marie-Antoinette qui constitue l'enjeu. L'épistolière formule donc avec force ses attentes à l'égard de sa partenaire: comme toute mère et épouse, Marie-Antoinette a le devoir de ramener au sein de la famille les membres qui s'en sont éloignés et risquent de se tourner contre elle. La figure qui renvoie à la sphère privée est complétée cependant par une figure qui renvoie à la sphère publique, celle de la reine médiatrice qui a des devoirs par rapport à la nation. Et c'est à cette face-ci que va la préférence d'Olympe, la sphère domestique (pour adopter les termes de Geneviève Fraisse) fournissant le modèle de la sphère publique dans laquelle l'intervention de Marie-Antoinette est sollicitée.

Deux buts s'inscrivent dans la lettre d'Olympe: amener la reine à faire revenir les princes émigrés fomentant une mutinerie contre la France révolutionnaire, et l'inciter à soutenir la cause des femmes, plus précisément à apporter son soutien à l'entreprise que constitue le lancement d'une nouvelle Déclaration des droits. La sollicitation politique cède ainsi la place à la demande de 
travailler à l'instauration de l'égalité entre femmes et hommes. Cette entreprise qui, aux dires d'Olympe, sera au profit de Marie-Antoinette, s'insèrera dans un projet plus vaste concernant toutes les femmes: ainsi se confirme le rôle éminent que l'épistolière entend réserver à la destinataire qu'elle souhaite associer à sa grande initiative. On voit qu'à partir du moment où Olympe donne le beau rôle à sa partenaire, la présentation de soi est relativisée, et l'équilibre entre les deux images rétabli. La conclusion de la lettre reprend les deux buts. Euvrer pour l'égalité du sexe féminin par rapport au sexe masculin, et travailler à la fois pour l'abandon de tous les privilèges sociaux et pour le ralliement du " parti monarchique » à la cause de la liberté. À la fin de son adresse à la reine, Olympe met curieusement la liberté tout autant en valeur que l'égalité qui, jusque-là, restait prépondérante. L'épistolière accentue à présent le rôle politique de sa destinataire. Cette accentuation sert cependant l'objectif premier d'Olympe. Si la reine révise son rôle politique et se fait tribun, l'initiative de l'auteure de la Déclaration aura de réelles chances d'aboutir.

L'injonction de défendre les intérêts du sexe féminin est placée au centre de la lettre; Olympe invite Marie-Antoinette à « donner du poids à l'essor des Droits de la Femme, et d'en accélérer les succès » (1993, p. 205). Ici, le discours politique est subverti par la revendication d'égalité. La reine doit prendre le parti des femmes et donner à son sexe « toute la consistance dont il est susceptible », car « cette Révolution ne s'opérera que quand toutes les femmes seront pénétrées de leur déplorable sort, et des droits qu'elles ont perdus dans la société ». Notons que ce constat n'est pas exempt de scepticisme. Tout semble alors dépendre de celle qui est épouse-mère et première dame de France à la fois, et cette double fonction la destinerait à prendre la tête des femmes. Considéré ainsi, l'argument ne perd rien de sa force, d'autant plus si l'on donne tout leur poids aux intimations - «Soutenez [...] Défendez [...] Croyez-moi [...]» et à la modalisation - «Voilà par quels exploits vous devez vous signaler [...] ». La figure de celle qui parle porte ici les traits du guide.

Que peut-on, dans cette lettre, observer dans le mode du dire? Si on prend le texte dans son ensemble, on relèvera le dialogue qui le sous-tend et qui, ponctuellement, devient explicite. Ainsi, «Oui, Madame» implique une réserve, une objection ou une interrogation venant de l'autre, et simule une interaction verbale de femme à femme où abstraction est faite du rang social et de la place qu'on occupe dans le champ politique. Par ailleurs, à observer les éléments constitutifs du discours, des implicites forts peuvent être relevés. Dans «celle que le hasard a élevée à une place éminente» est sous-entendu que tous sont égaux, toutes sont égales, les places apparaissant comme interchangeables: Olympe pourrait être à la place de la reine et vice versa. L'égalité devient un présupposé crucial, indépassable. Ajoutons que, dans la clôture, apparait la figure de la citoyenne modèle susceptible d'être imitée par la reine elle-même: «Je perds le but de cette dédicace. C'est ainsi que tout bon citoyen 
sacrifie sa gloire, ses intérêts, quand il n'a pour objet que ceux de son pays. » Si l'écriture tâche d'équilibrer la cause de l'égalité des sexes et celle de la liberté, elle instaure cependant, dans le mode du dire, un rapport de femme à femme.

On voit que le texte tend à une subversion globale : à la fois sur le plan des prescriptions rhétoriques et sur celui des normes sociales, à la fois dans la présentation du champ politique et de celui des relations entre les sexes. Qui plus est : ne se trouve-t-on pas, ici déjà, dès avant la Déclaration des droits de la fermme, face à la position la plus avancée? On est tenté de dire que s'y dessine moins l'idée d'une fraternité incluant les femmes que l'idée de la sororité qui, quant à elle, aurait pu désigner « un lien nouveau entre les femmes, utopie d'un lien supérieur à celui des hommes entre eux» (Fraisse, 2000, p. 81).

\section{Au Tribunal révolutionnaire}

En septembre 1793, Madame de Gouges, incarcérée à l'Abbaye de SaintGermain-des-Prés, réussit à rédiger et à faire diffuser une adresse Au Tribunal révolutionnaire ${ }^{5}$ :

Tribunal redoutable, devant lequel frémit le crime et l'innocence même, j'invoque ta rigueur, si je suis coupable. Mais écoute la vérité:

L'ignorance et la mauvaise foi sont enfin parvenues à me traduire devant toi : je ne cherchais pas cet éclat. Contente d'avoir servi dans l'obscurité la cause du Peuple, j'attendais avec modestie et fierté une couronne distinguée que la postérité seule peut donner à juste titre à ceux qui ont bien mérité de la patrie.

Ce texte destiné à être affiché sur les murs de Paris se présente comme un plaidoyer. Or le choix du support suffit en lui-même à subvertir le genre. À travers lui, en effet, un dispositif d'énonciation lié à la procédure judiciaire et à la salle d'audience s'ouvre à un public potentiel plus large. En même temps, ce dispositif est au sens strict porté sur la place publique.

La subversion du plaidoyer, cependant, ne s'arrête pas là. Et cela pour deux raisons essentielles. Tout d'abord, Olympe passe à plusieurs reprises de l'acte de défense à l'acte d'accusation. On lit ainsi dès le troisième paragraphe:

Pâlissez, vils délateurs, votre règne passe [...]. Vieux esclaves des préjugés de l'Ancien Régime [...], républicains de quatre jours, il vous sied bien d'inculper une femme née avec un grand caractère et une âme vraiment républicaine. Les taches que vous avez imprimées à la Nation française ne peuvent être lavées que par votre sang.

5. Affiche signalée par Latour-Lamontagne dans son rapport de police du 21 septembre $1793-$ Écrits politiques 2 (p. 254 et suiv.). 
Le choix d'un genre destiné à la défense n'empêche pas la plaignante d'adopter une attitude offensive. Par ailleurs, la locutrice s'adresse, au fil de son argumentation, à des instances diverses : aux magistrats d'abord, aux membres du Comité de salut public ensuite - «Frémissez, Tyrans modernes!» -, aux «Républicains» (magistrats et public réunis) et aux « Français » (le public sans les magistrats), enfin à « toi, mon fils » (le jeune Aubry, général de l'armée républicaine). Ainsi, le public est invoqué comme instance de légitimation et apparait, côte à côte avec les juges du tribunal révolutionnaire, comme un allocutaire à part égale - et même supérieur puisque les «Français » sont désormais considérés comme le véritable magistrat, au-dessus du tribunal :

Ah! Français, je ne peux me rappeler ce traitement sans verser des larmes. Vous aurez de la peine à croire que des hommes, des magistrats soi-disant populaires, aient poussé la férocité jusqu'à me refuser pendant sept jours de faire appeler un médecin.

Cette affiche et mon mémoire [projet d'un référendum occulté par le Comité de salut public] vont, par le moyen de la distribution à la main, éclairer le public; oui, mes concitoyens, ce comble d'iniquité va servir mon pays.

La place des magistrats est relativisée et, qui plus est, un décalage est marqué entre le tribunal et le public.

Cette relativisation se retrouve sous une autre forme à la fin du texte d'Olympe qui assigne à son propre fils la place jusque-là réservée au public: «Et toi, mon fils, de qui j'ignore la destinée, viens en vrai Républicain te joindre à une mère qui t’honore [...]. Viens en vrai Républicain demander la loi du Talion contre les persécuteurs de ta mère ! » Notons que c'est cette allocution personnelle, rattachant la renommée publique à la relation privée, qui marque la clôture de ce texte. Ainsi l'image de soi qui, jusque-là, a réuni deux faces, la servante anonyme de la nation et la femme au grand caractère, est complétée par la figure de la mère. Dès lors qu'on la considère dans son ensemble, l'adresse publique d'Olympe apparait comme placée entre l'invocation à la justice qu'autorisait la situation de l'accusée, et l'affirmation de la mère fière et sensible qui sollicite l'intervention de son fils.

La subversion du genre s'effectue ici, cependant, dans des circonstances très particulières, en vue d'obtenir gain de cause dans un procès qui menace la vie de la plaignante. On comprend donc qu'avec Au Tribunal révolutionnaire, Madame de Gouges change de registre. Ce n'est plus l'enjeu de l'égalité entre les sexes qui est mis en avant ; au détriment de celui-ci s'impose à présent l'argument politique (au sens premier de ce terme). L'autoportrait qui se dessine dans l'adresse aux magistrats ne montre pas celle qui défend la cause des femmes mais celle qui sert la cause du peuple. Sur le plan de l'argumentation, les traits mis en valeur servent à opposer la plaignante à ses ennemis, qui sont par là même inculpés. Deux forces, deux intérêts politiques sont 
placés face à face - républicanisme et anarchie, liberté et despotisme. Ces forces antagonistes sont considérées comme tributaires de dispositions inconciliables - amour pour le peuple versus calomnie, défense de l'honneur de la nation versus soif de sang. Mais il y a plus: les magistrats d'une part, le public d'autre part, sont invités à prendre parti. Ce que le texte suggère à ses lecteurs potentiels, c'est de donner raison aux "vrais soutiens de la patrie» aux dépens des faux républicains qui complotent contre la nation, et à la «conscience pure et imperturbable» aux dépens de «l'ignorance et la mauvaise foi $»$.

L'inflexion du discours de l'égalité dans le sens du discours de la liberté ne peut guère être expliquée sans le recours à la situation qui, en septembre 1793, caractérise à la fois la politique française et le statut public et privé de Madame de Gouges. Au moment où l'existence des clubs de femmes est menacée, cette intervention fait partie du projet jacobin d'écarter les femmes de la politique. Plus précisément, il règne, après l'exécution de Charlotte Corday, un climat de suspicion. "L'effervescence, savamment entretenue dans les discours et les feuilles démagogiques, annonça la traque aux "suspects" » (Blanc, 2003, p. 201). C'est dans ce contexte qu'on doit situer l'arrestation de Madame de Gouges comme auteur du placard Les Trois Urnes ou le salut de la patrie par un voyageur aérien, qui propose d'organiser un vote dans chaque département sur la forme du gouvernement à établir, républicain, fédéraliste ou monarchique (juillet 1793). Après un premier interrogatoire, elle est mise au secret par Marino, l'administrateur du Comité de salut public, un proche de Robespierre qu'elle présente comme son ennemi déclaré. La référence à Cubières, son ami qui écrit pour la Feuille du Salut Public, ne l'aide pas, et la lettre qu'à la première occasion elle rédige à son adresse est interceptée. On se rend compte combien, dans une telle conjoncture, la démarche d'Olympe est audacieuse lorsque, deux mois plus tard, en septembre, elle tâche de s'adresser aux Parisiens par voie d'affiche. Comme Olivier Blanc le dit avec pertinence: «Ne reniant pas ses opinions de républicaine humaniste, attachée aux Droits de l'Homme, elle faisait preuve d'une pugnacité rare, héroïque» (2003, p. 212).

Dans ces circonstances, Madame de Gouges se voit tenue de se placer sur le plan politique seul. Dans quelle mesure son argumentation implique-t-elle néanmoins l'enjeu égalitaire? Sans doute trouve-t-on des désignations telles que «soutiens de la patrie » ou «ceux qui ont mérité de la patrie», qui ne font pas de distinction de sexe. Mais on a, par ailleurs, pu relever la figure de la femme libre confrontée à celle de l'homme esclave, ou de la femme patriote confrontée au despote. Ainsi, dans «c'est avec les armes du courage et les armes de la probité que je vous demande compte de la tyrannie que vous exercez sur les vrais soutiens de la patrie [...]; j'ai bravé les sots, j'ai frondé les méchants», le courage et la probité sont mis en relief comme qualités qui 
distinguent la femme politique des hommes qui la persécutent. II faut ajouter que, lorsque Madame de Gouges caractérise ces qualités, l'image qu'elle donne d'elle-même associe étroitement sphère privée et espace public: "Vous me forcez à tirer vanité de ces avantages, dons précieux de la nature, de ma vie privée et de mes travaux patriotiques. " Et elle poursuit: «Les taches que vous avez imprimées à la Nation française ne peuvent être lavées que par votre sang que la loi fera bientôt couler sur l'échafaud. " La figure allégorique de la Nation est ainsi rapprochée de la locutrice, qui se donne à voir en «femme née avec un grand caractère et une âme vraiment républicaine», comme si Olympe entendait, sous l'égide de la haute instance de la Nation, affirmer sa propre supériorité sur les hommes qui tombent dans la lâcheté et le despotisme. Ceci implique qu'elle prétend remplir en tant que femme la fonction dont les hommes s'efforcent de garder le monopole, et d'y parvenir mieux qu'ils n'ont su le faire. La prétention à un statut élevé de la femme résonne encore dans l'intimation et l'assertion suivantes: «Vous, magistrats [...], apprenez à me connaitre! Ennemie de l'intrigue, loin des systèmes, des partis [...], je me suis frayé une route nouvelle.» Enfin, l'enjeu égalitaire, "féministe», est implicite également dans les diverses facettes qui constituent l'image de la locutrice: la mère patriote qui honore son fils et qui sollicite son secours, la femme qui se bat pour la liberté et défend la cause du peuple, mais aussi la rédactrice de la Déclaration des droits de la femme et de la citoyenne ${ }^{6}$.

En effet, ce texte explosif d'Olympe de Gouges constitue un intertexte important de l'adresse Au Tribunal. Ainsi, «J'invoque la rigueur si je suis coupable » fait écho aux articles VII et X de la Déclaration des droits dédiée à la reine: "Les femmes obéissent comme les hommes à cette loi rigoureuse», et: «La femme a le droit de monter sur l'échafaud; elle doit avoir également celui de monter à la tribune. »Égalité négative et positive: pour celle-ci, il faut remarquer que l'article VI précise que «toutes les citoyennes et citoyens [sic] doivent [...] concourir à la formation [de la loi]». Madame de Gouges réclame l'égalité comme due aux femmes de par la nature et la raison, tout en militant pour qu'elles accèdent pleinement aux droits politiques, "actifs », que Sieyès et les Jacobins leur refusent, ne leur concédant que les droits naturels et civils, «passifs».

À travers cette argumentation et ces images de la femme, les lecteurs potentiels sont invités à considérer Olympe comme femme qui défend les intérêts de la nation et que les hommes de la fraction radicale tâchent de détruire. Cette femme est érigée en exemple, placée au même niveau que les magistrats et au-dessus des Jacobins. En d'autres termes, le statut réclamé ouvertement dans la Déclaration est ici attaché à la femme implicitement, dans le

6. Pour les images de la femme dans le discours politique de l'époque, voir D. Godineau (2001). 
dire. C'est dans cette perspective que la subversion du genre se relie, malgré les apparences, au discours égalitaire. On a vu, en effet, que le plaidoyer adressé à une instance constituée exclusivement d'hommes tourne à l'acte d'accusation - et, plus précisément, que le plaignant est une femme qui, face à cette instance masculine, se dresse contre un autre groupe d'hommes, les robespierristes, qu'elle dénonce comme étant à la source de l'accusation dressée contre elle. On conclura donc que la locutrice cherche à redistribuer les places en changeant l'ordre prescrit par les hommes. C'est dans ce sens que Au Tribunal révolutionnaire fait entrevoir une femme qui occupe une position pour le moins égale à celle du sexe masculin, et qu'il rappelle la revendication d'Olympe de Gouges femme et citoyenne.

Dans quelle mesure chacun des trois textes donne-t-il à voir le projet de l'instauration de l'égalité entre les sexes? Dans La Lettre au peuple, l'égalité se dessine plutôt en filigrane, et, dans la clôture, elle a la fraternité pour complément. La locutrice se présente comme une rhétoricienne capable de monter à la tribune tout autant que l'homme, et dotée d'un savoir équivalent au sien; la citoyenne ingénieuse et zélée va jusqu'à se placer au même rang que le roi. Dans le deuxième texte, l'Épître à la reine, à qui elle demande de l'aider au lancement d'une nouvelle Déclaration des droits centrée cette fois sur les femmes, Olympe prétend à une place équivalente à celle de sa destinataire. Elles doivent toutes deux s'associer dans la lutte qui les oppose aux hommes pour réaliser le projet égalitaire. Dans cette entreprise qui serait menée par deux femmes, on peut déceler l'utopie de la sororité. Le discours sur les sexes prédomine, et l'accent est mis sur la cohésion du «parti » des femmes. Enfin, dans le troisième texte, l'adresse publique Au Tribunal révolutionnaire, l'argument politique prévaut. Sans doute Olympe, à la même époque, rappelle-t-elle la revendication féminine et la solidarité entre femmes qui conditionne celleci : «Les femmes seront-elles toujours isolées les unes des autres, et ne ferontelles jamais corps avec la société, que pour médire de leur sexe, et faire pitié à l'autre? »7 Mais, dans l'adresse Au Tribunal, la revendication de l'égalité des sexes semble reléguée à l'arrière-plan. Si la profonde préoccupation de Madame de Gouges réapparait néanmoins, c'est dans une image singulière qui finit par imprégner le texte: celle d'une lutte à mort entre des hommes et une femme qui entend agir dans l'espace public. Ainsi, en quelque sorte, en dépit de sa situation et des considérations stratégiques qui l'amènent à privilégier l'argument politique au détriment de la différence des sexes, son intérêt premier explicité dans l'Épître à la reine réapparait jusque dans son plaidoyer politique.

À comparer les trois textes, on perçoit le tournant que constitue l'automne 1793. L'égalité sous ses diverses formes devient, de principe fondamental, un

7. Cité par G. Fraisse, 2000, p. 81. 
trait d'arrière-plan, ce qui s'explique par la conjoncture politique. La femme politique influente est à présent dénoncée et traitée comme contre-révolutionnaire par les Jacobins. Or, on a pu voir que celle qu'on désigne comme ennemie de la République est en réalité l'adversaire des hommes qui considèrent leur pouvoir comme mis en cause par les femmes. C. Veauvy et L. Pisano parlent à juste titre de l'expérience de la frontière et de la transgression d'Olympe. C'est cette expérience traduite en acte qui a dû être perçue comme une provocation et une menace par les hommes lorsqu'ils ont brutalement raffermi l'inégalité des sexes.

\section{Références}

BLANC 0., 2003, Marie-Olympe de Gouges, une humaniste à la fin du XVIII ${ }^{e}$ siècle (s. I.), Belaye, Éditions René Viénet.

FRAISSE G., 2000, Les deux gouvernements : la famille et la Cité, Paris, Gallimard.

GODINEAU D., 2001, "Beauté, respect et vertu: la séduction est-elle républicaine?

(1770-1794)», C. Dauphin et A. Farge éd., Séduction et société. Approches historiques, Paris, Le Seuil, p. 89-121.

GOUGES O. (de), 1788-1791, Écrits politiques 1, 1792-1793, Écrits politiques 2, préface d'O. Blanc, Paris, Côté-femmes.

PLANTÉ C. éd., 1998, L'épistolaire, un genre féminin ? Paris, Champion.

SIESS J. et VALENCY G. éd., 2000, La double adresse, Paris, L'Harmattan.

VEAUVY C. et PISANO L., 1997, 1997, Paroles oubliées. Les femmes et la construction de l'État-nation en France et en Italie, 1789-1860, 1789-1860, Paris, Colin. Traduit de l'italien, 1994, Parole inascoltate. Le donne e la costruzione dello Stato-nazione in Italia e in Francia, 1789-1860, Testi e documenti, prefazione di G. Conti Odorisio, Roma, Editori Riuniti. 